\title{
Alguns aspectos sobre morfologia e ciclo de vida de Limnogonus recurvus (Hemiptera: Gerridae)
}

Bento Melo Mascarenhas ("*)

\begin{abstract}
Resumo
Alguns aspectos da biologia de Limnogonus recurvus (Gerridae: Hemiptera) foram estudados no laboratório. Os adultos e ninfas foram capturados em vários tipos de habitats aquáticos, como: rios, igarapés, lagos e pequenas poças de água pluvial. Os cruzamentos foram efetuados no laboratório. O ciclo de ovo a adulto é de aproxidamente 43 dias.
\end{abstract}

\section{INTRODUÇÃo}

Este trabalho se propõe a mostrar aigumas considerações sobre a morfologia e ciclo de vida de Limnogonus recurvus Drake \& Harris. 1930 (Gerridae: Hemiptera). Esta espécie é bem comum na região Amazônica. Encontramos exempiares desta espécie em igarapés, tanto de água preta como de água branca, bem como em lagos e poças formadas por água de chuva. Adapta-se facilmente ao regime de cativeiro. O seu hábito alimentar não é exigente Sua grande longevidade contribui para que se reaiizem, em laboratório, observações e experiências relacionadas à sua biologia .

\section{REVISÃo BIBLIOGRÁFICA}

$\mathrm{Na}$ sistemática dos hemípteros, a família Gerridae, está relativamente bem estudada no Brasil. Na região Amazônica os gerrídeos estão bem conhecidos taxionomicamente, graças aos trabalhos de: Drake \& Harris (1930), Shaw (1933), Kenaga (1941, 1942), Kuitert (1942), Drake \& Carvalho (1954), Drake \& Chapman (1954) e Drake $(1954,1957$ e 1959). Estes trabalhos limitam-se a descriçōes de gêneros, espécies e subespécies.

A familia Gerridae foi descrita, pela primeira vez, em 1807 por Leach; posteriormente, em 1843, Amyot e Serville fizeram uma nova descrição. Compreende 5 subfamilias: Rhagadotarsinae Lundblad, Ptilomerinae Bianchi, Trepobatinae Matsuda, Gerrinae Amyot e Serville e Halobatinae Bianchi (Poisson, 1965). Segundo Matsuda (1960), a subfamília Gerri. nae apresenta 4 tribos: Gerrini com 10 gêneros e 8 subgêneros (é nesta tribo que se encontra o gênero Limnogonus); Cylindrostethini com 3 gêneros; Charmatometrini com 3 gêneros e Eotrechini com 5 gêneros; a famíiia toda está constituída por 53 gêneros e 16 subgêneros. Os gerrídeos da América Meridional pertencem às subfamilias Gerrinae e Halobatinae (Costa Lima, 1940).

Os ovos de Gerris são depositados sobre suportes no interior da água ou sobre objetos flutuantes abaixo da superfície da água; são fixados nestes substratos por tima substância gelatinosa impermeável à água (Dufour, 1833) Estes ovos são de forma cilíndrica, arreciondados nas extremidades e ligeiramente côncavos na extremidacie micropilar; não possueın opérculos e o cório é relativamente espesso em suas extremidades (Torre-Bueno, 1917). Em virtude destes ovos não possuirem opérculo, a área angular anterior do uvo fende-se para a eclosão da ninfa; forma-se entre os olhos uma linha negra brilhante chamada de linha de fraqueza ou de ruptura. O cório nesta região é fino e fraco, e a direção e extensão da ruptura é constante (Tonapi, 1959).

Trabalhos de morfologia interna e exierna da família Gerridae, foram feitos por vários autores. Poisson (1922) descreveu a yenitá. lia do macho do gênero Gerris baseado em: Gerris (Hygrotrechus) paludum Fabricius, Ger. ris (Limnotrechus) gibbifera Schummel, G. (L) argentatus, $G$. (L) costae (Herrich - Schaffer). G. (L) thoracicus Schummel, G. (L) lacustris,

\footnotetext{
(*) - Primeira pérte da tese apresentada ao Curso de Pós-Graduaçăo do Instituto Nacional de Pesquisas da Amazônia (INPA) e Fundação Universidade do Amazonas (FUA), para a obtenção do grau de Mestre em Ciências.

(*") - Museu Paraense Emílio Goeldi, Belém.
} 
G. (L) odontogaster e G. najas (De Geer). Schoeder (1931), descreveu a genitália de algumas espécies do gênero Rheumatobates. Matsuda (1960) descreve aspectos de morfologia dos gerrídeos de várias partes do mundo. Darnhofer-Demar (1969) descreve a morfolo. gia do aparelho locomotor de Gerris lacustris

A duraçấo do ciclo de vida de várias espécies de Gerris apresentam algumas variações. O Gerris remiges Say, apresenta um ciclo de vida de 46 dias (Torre-Bueno, 1917); o Gerris conformis (Uhler), 34 dias (Hunger. ford, 1919); o Gerris notabilis Drake \& Hottes, 43 dias e o Gerris gillettei Lathiery \& Serevin, 28 dias (Callahan, 1974) .

Vários trabalhos relacionados com as formas imaturas, especialmente dentro do gênero Gerris, foram feitos na Europa e nos Estados Unidos. A descrição da ninfa de Gerris fluviorum Fabricius, foi feita por Tonapi (1959). As ninfas de Gerris dissortis Drake \& Harris, G. conformis, G. argenticolis Parshley, G. canaliculatus Say, G. buenoi Kirkaldy, G. comatus Drake \& Hottes, G. marginatus, 'G. insperatus Drake \& Hottes e G. remiges, da Nova Inglaterra, Estados Unidos, foram estudados taxionomicamente por Sprague (1967); o trabalho baseou-se fundamentalmente nas medidas das ninfas, forma e cor básica, que são caracteres úteis para a determinação de espécies de Gerris. Os trabalhos de Poisson (1957) e Brinkhurst (1959), dão figuras e chaves para a determinação das ninfas de Gerris, para as espécies da França e Inglaterra, respectivamente. Hoffman (1924), estudou Gerris buenoi. Muito embora seu trabalho não tenha figuras, a descrição do quarto estágio ninfal é pormenorizada e inclui o modelo do abdome; a descrição do quinto estágio é incompleta. Penn \& Goldsmith (1950) e Bobb (1951), estudaram a biologia de Gerris canaliculatus durante um ano, fazendo medidas do corpo e das pernas. O dimorfismo sexual é muito evidente. Os machos adultos atingem cerca de $3 / 4$ do comprimento das îêmeas. Estas diferenças são aparentes também, no comprimento das pernas no quinto estágio ninfal; neste estágio, as pernas idos machos são maiores que as das fêmeas. Os desenhos de vários estágios dados no trabaiho de Bobb, são úteis para a separação dos vários estágios um dos outros.
Hoffman (1924) descreveu G. (limnoporus) dissortis, o qual foi considerado sendo o mesmo da Europa G. (Limnoporus) rufuscutellatus Latreille. As medidas de Hoffman foram mais apropriadas para o $1^{\circ}, 2 .^{\circ}$ e $3 .^{\circ}$ estágios ninfais. ficando o $4 .^{\circ}$ e $5 .^{\circ}$ estágios com uma descrição deficiente. Mencionou que a cor básica para estes estágios é a marrom, porém não mencionou o modelo listrado que é evidente para o $5^{\circ}$ estágio ninfal (Sprague, 1967).

\section{MATERIAL E MÉTODOS}

MATERIAL - Foram usados ovos, ninfas e adultos de Limnogonus recurvus (Gerridae: Hemiptera) .

\section{MÉTODOS}

1 - Coleta - O material para criação em laboratório foi coletado em lago artificial no setor residencial do INPA (Instituto Naciona! de Pesquisas da Amazônia) Manaus-Amazonas, agosto de 1977, com uma rede entomológica aquática.

\section{2 - Criação de L. recurvus no laboratório.}

Foram utilizados apenas dois casais macrópteros. Estes foram trazidos do lago já referido anteriormente, ao laboratório em uma cuba plástica transparente coberta com uma tela de nailon com malha fina, presa com elástico comum. No laboratório cada casal foi colocado em uma cuba, onde houve cópula normal e fertilização dos ovos. A cada 24 horas eram retirados os ovos, contados e separados em cubas plásticas menores. Para tal experiência, foram usadas cubas plásticas de tamanhos: $25,5 \times 14,5 \times 6,5 \mathrm{~cm}, 19 \times 10 \times 6,5 \mathrm{~cm}$, $10 \times 10 \times 6,5 \mathrm{~cm}, 20,5 \times 14 \times 6,5 \mathrm{~cm}, \quad 18 \times$ $11 \times 6,5 \mathrm{~cm}$ e $11 \times 9 \times 6,5 \mathrm{~cm}$. As cubas foram ccbertas com tela de maiha fina, presa com elástico. Foram cheias com uma camada de água de torneira até uma altura de aproximadamente $3 \mathrm{~cm}$. Além da água, foi introduzida vegetação aquática na cuba, das espécies Salvinia auriculata Aublet, Cabomba aquática Aubiet e raizes de Eichhornia crassipes (Mart.) Solms, para servir como um substrato para oviposição e fixação dos ovos. Algumas vezes, propositadamente não foi introduzido nas placas vegetação flutuante. 
No princípio, após a eclosão dos ovos, as ninfas eram conservadas em suas cubas. De pendendo do número de ninfas nascidas, utilizaram-se cubas de tamanho adequado, com o mesmo sistema de proteção, também referido. Posteriormente, em virtude do grande índice de mortalidade decorrente do canibalismo das ninfas e por não se poder fazer um melhor controle de seu cicio de vida, as ninfas de cada postura foram criadas separadamente em copos plásticos de dois tamanhos: o maior tinha $9 \mathrm{~cm}$ de altura e o menor $7,5 \mathrm{~cm}$ de al. tura, o diâmetro de ambos $6,5 \mathrm{~cm}$. Os copos foram cheios com $2 / 3$ de água e cobertos com teia de nailon de malha fina, presa com elástico.

A água dos copos não foi trocada durante todo o crescimento da ninfa, ou seja, do primeiro ao quinto estádio. Com uma pequenina rede de arame de forma circular, a cada 24 horas, o alimento do dia anterior era retirado (no caso insetos já em decomposição), bem como as exúvias quando existiam e algumas partículas de detritos que conseguiam passar através da tela.

\section{RESUltados}

\section{OVIPOSIÇÃo}

Foi observado, no laboratório, que as têmeas de L. recurvus depositam seus ovos num substrato qualquer submerso. Foi verificado, na vegetação flutuante utilizado como substrato para a fixação dos ovos, o seguinte: nas raizes de Eichhornia crassipes os ovos eram distribuídos desordenadamente, na Cabomba aquática a distribuição já era um pouco mais uniforme e em Salvinia auriculata os ovos foram depositados uniformemente na face dorsal da folha, obedeciam a organização de uma fila indiana, colocados lado a lado. Observou-se que na ausência de um substrato apropriado, estas fêmeas fixam seus ovos nas paredes das placas. Outras vezes, foram notados ovos fixados aos cadáveres de insetos utilizados como alimento e nesta situação, há alteração no tempo de incubação destes, chega quase a duplicar. Não foram observados ovos soltos flutuando ou submersos na água das placas. $\mathrm{O}$ número de ovos postos por fêmea variou consideravelmente; na tabela 1 temos o número de ovos eliminados em 7 dias por 15 fêmeas.

\section{MORFOLOGIA DO OVO}

A forma e o tempo de morfogênese são mostrados na fig. 1.

\section{ECLOSÃO DO OVO E DESENVOLVIMENTO DA NINFA}

Foram observados ao microscópio por transparência, jovens em eclosão de 10 espécimes de $L$. recurvus.

Foi notado que momentos antes da eclosão, um certo movimento do abdome da ninfa, no mesmo instante em que aumenta gradativamente o volume da região cefálica do ovo. Iniciou-se a rupiura do cório na região correspondente à linha de fraqueza, situada entre os olhos. A ninfa escapou do ovo por esta fenda que se abriu, exteriorizou o rosto acompanhado pela cabeça, ainda envoltos pela membrana vitelínica; executou movimentos para frente e para trás libertando o resto do corpo, estes movimentos são lentos e rítmicos; à medida que projetou seu corpo para fora do ovo, o ângulo formado por ela em relação ao mesmo foi de aproximadamente $90^{\circ}$. Quando a membrana se rompeu e suas pernas anteriores se livraram, as outras partes do corpo sairam mais rapidamente. Para livrar as per-

TABELA 1 - Número de ovos postos por 8 fêmeas apteras e 7 fêmeas macrópteras em 7 dias.

\begin{tabular}{l|cl|c}
\hline $\begin{array}{c}\text { Fêmeas } \\
\text { ápteras }\end{array}$ & N.o de ovos & $\begin{array}{c}\text { Fêmeas } \\
\text { macrópteras }\end{array}$ & N.o de ovos \\
\hline primeira & 19 & primeira & 21 \\
segunda & 25 & segunda & 13 \\
terceira & 39 & terceira & 97 \\
quarta & 89 & quarta & 15 \\
quinta & 44 & quinta & 49 \\
sexta & 48 & sexta & 45 \\
sétima & 20 & sétima & 26 \\
oitava & 75 & & \\
\hline
\end{tabular}

O teste de Mann Whitney aplicado aos dados da Tabela acimo não revelou diferença significativa a nivel de $5 \%$ num teste bilateral. Isto implica que na semana que o experimento foi levado a efeito as fèmeas observados colocaram um número de ovos sem diferença paro o caráter áptero e macróptero. 
A

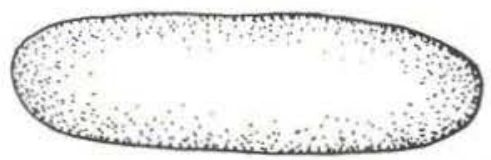

B

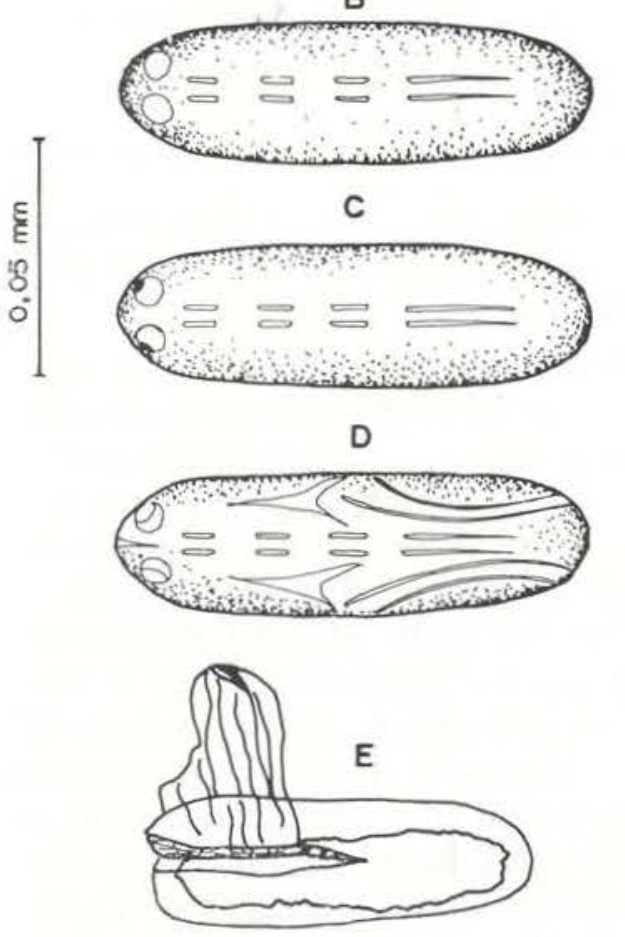

120 à 144

168 ò 192

Fig. 1 - Ovos de L. recurvus em várias fases de desenvolvimento (esquemático).

nas medianas e posteriores, a ninfa se inclina para trás. Neste processo de emergência a ninfa gasta aproximadamente 8 minutos. Ao emergir e libertar-se da membrana envolvente. a ninfa tenta, de imediato, atingir a superfície da água, através de impulsos do corpo para a frente, com as pernas totalmente estendidas; algumas vezes conseguem chegar, mas, na grande maioria das vezes, não; desta forma, ficam no fundo do corpo de água com as pernas totalmente estendidas e paradas, como se fossem uma estrela no fundo do recipiente; podem ficar submersas por algumas horas, mas sempre estão tentando alcançar a superfície; muitas vezes, depois de longas tentativas o conseguem, mas se não o conseguirem, em aproximadamente 8 horas, morrem na posição já reterida.

Após atingirem a superfície da água, executam movimentos de limpeza do corpo e an. dam ativamente a procura de alimento. Nesta idade de sua vida, foram utilizados como alimentos pequenos Hymenoptera da família Formicidae $\epsilon$ pequenos Diptera da família Drosophilidae.

A separação das ninfas em copos isolados, permitiu um melhor controle na alimentação; Foi verificado que $70 \%$ das ninfas fazem ecdise durante a noite e $30 \%$ durante o dia. No laboratório, foi verificado que, tanto os adultos como as ninfas de L. recurvus, são inativos durante a noite, ficando parados sobre a vegetação flutuante ou na superfície da água. Dados sobre a muda e $L$. recurvus são mostrados na tabela 2 e fig. 2.

TABELA 2 - Média do tempo (em dias), desvio padrăo, tempo máximo e tempo minimo, utilizado pelas ninfas de L. recurvus para fazerem a ecdise.

\begin{tabular}{c|c|c|ccc}
\hline $\begin{array}{c}\text { Estádio } \\
\text { ninfal }\end{array}$ & $\begin{array}{c}\text { N.o de ninfas } \\
\text { observadas }\end{array}$ & $\bar{x}$ & DP & \multicolumn{2}{|c}{ Dias } \\
\hline 1.0 & 173 & 6.0 & 1.52 & 10 & 4 \\
$2 .^{\circ}$ & 166 & 5.8 & 1.30 & 10 & 4 \\
$30^{\circ}$ & 166 & 5.8 & 1.12 & 10 & 4 \\
$4.0^{\circ}$ & 165 & 6.7 & 1.61 & 12 & 4 \\
$5 .^{\circ}$ & 156 & 9.2 & 1.19 & 15 & 6 \\
\hline
\end{tabular}

\section{ESTÁGIO NINFAL}

Os estágios ninfais são descritos neste trabalho pela primeira vez. Na tabela 3 , são mostradas as principais características para identificar tais estágios. Dados biométricos das ninfas e adultos são mostrados na tabela 4. Nas figuras $2 A, 2 B, 2 C, 2 D$ e $2 E$, estão ilustradas as ninfas de $1 .^{\circ}$ a $5 .^{\circ}$ estádios.

\section{MORTALIDADE NO LABORATÓRIO}

As possiveis catisas da mortalidade verificada em laboratório, principalmente para o $1^{\circ}$ estádio ninfal, são a formação de uma fina película de bactérias na superfície da água e a incapacidade de algumas ninfas em alcançar a superfície da água por seus próprios meios logo após o nascimento. Este parece ser $\alpha$ fator mais importante neste estádio, cuja taxa de mortalidade chegou a $23 \%$. As outras mor- 


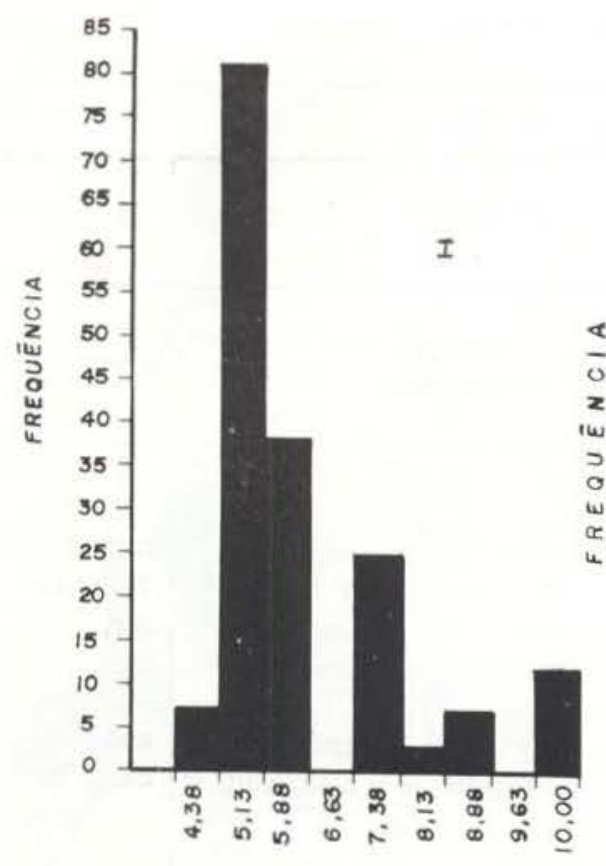

DIAS

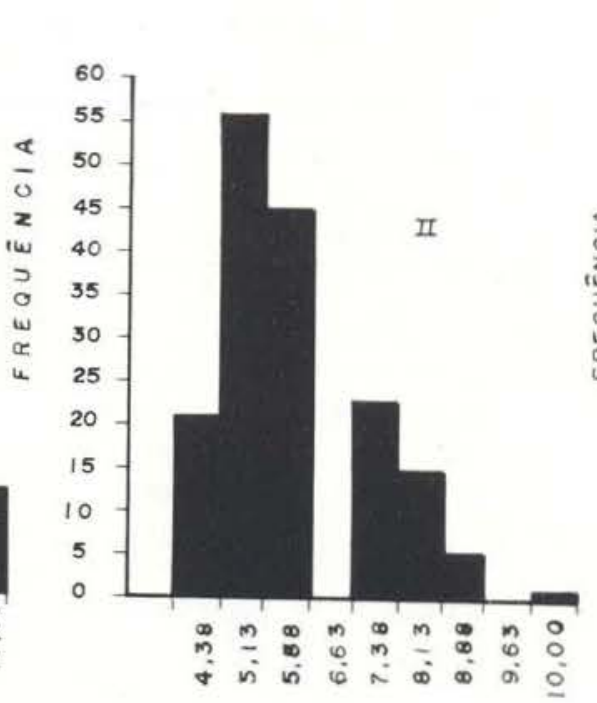

DIAS

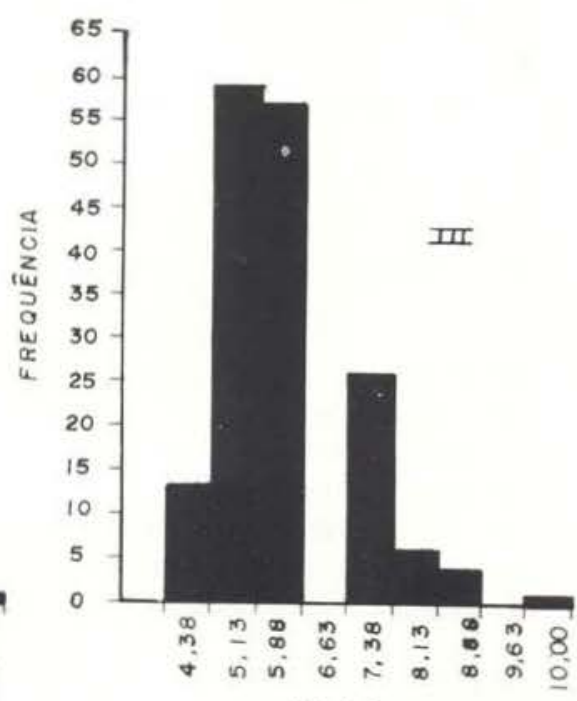

DIAS
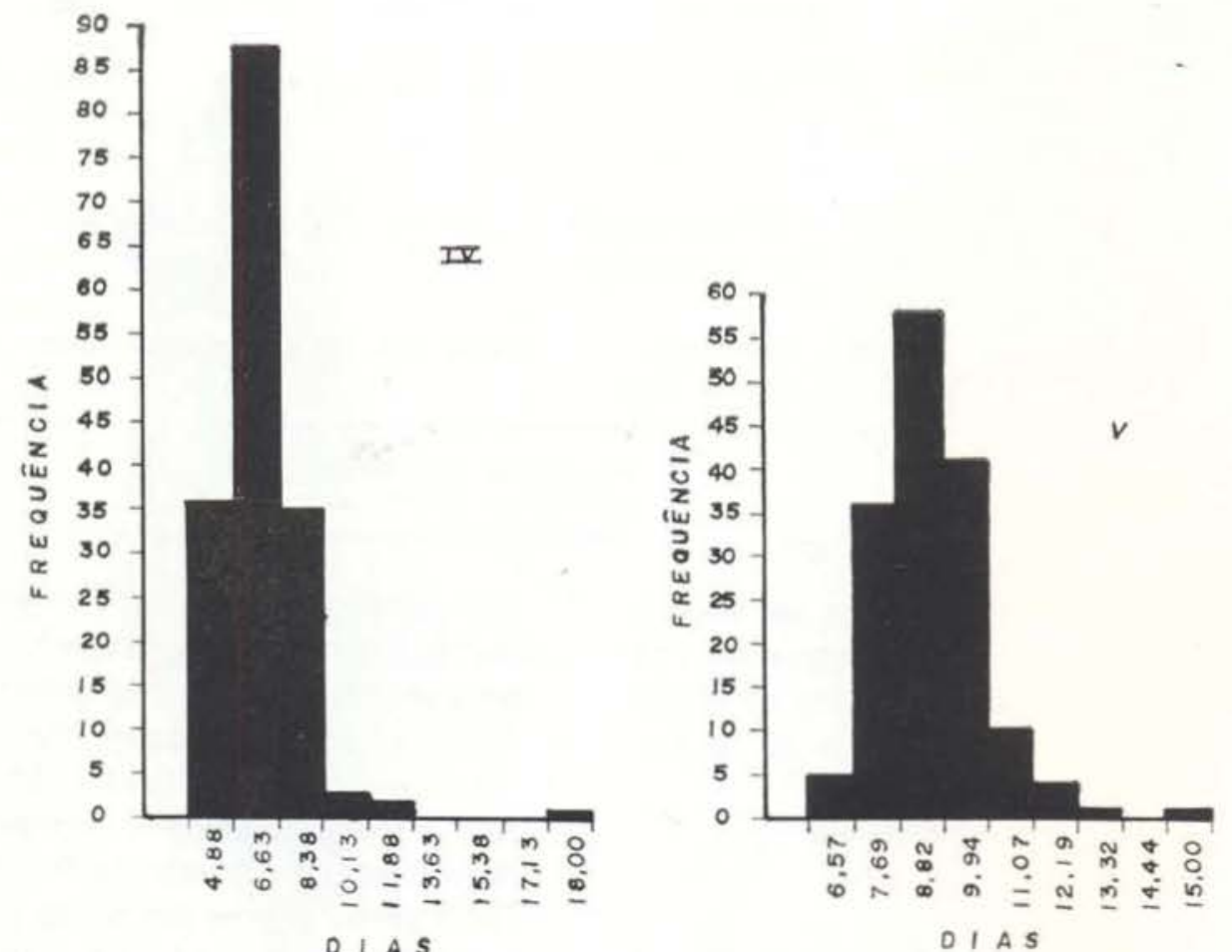

Fig. 2 - Mudanças de estágio ninfal em L. recurvus. I - primeira muda; II - segunda muda; III - terceira mu da; IV - quarta muda; V - quinta muda. 
TABELA 3 - Características básicas para identificar ninfas de Limnogonus recurvus

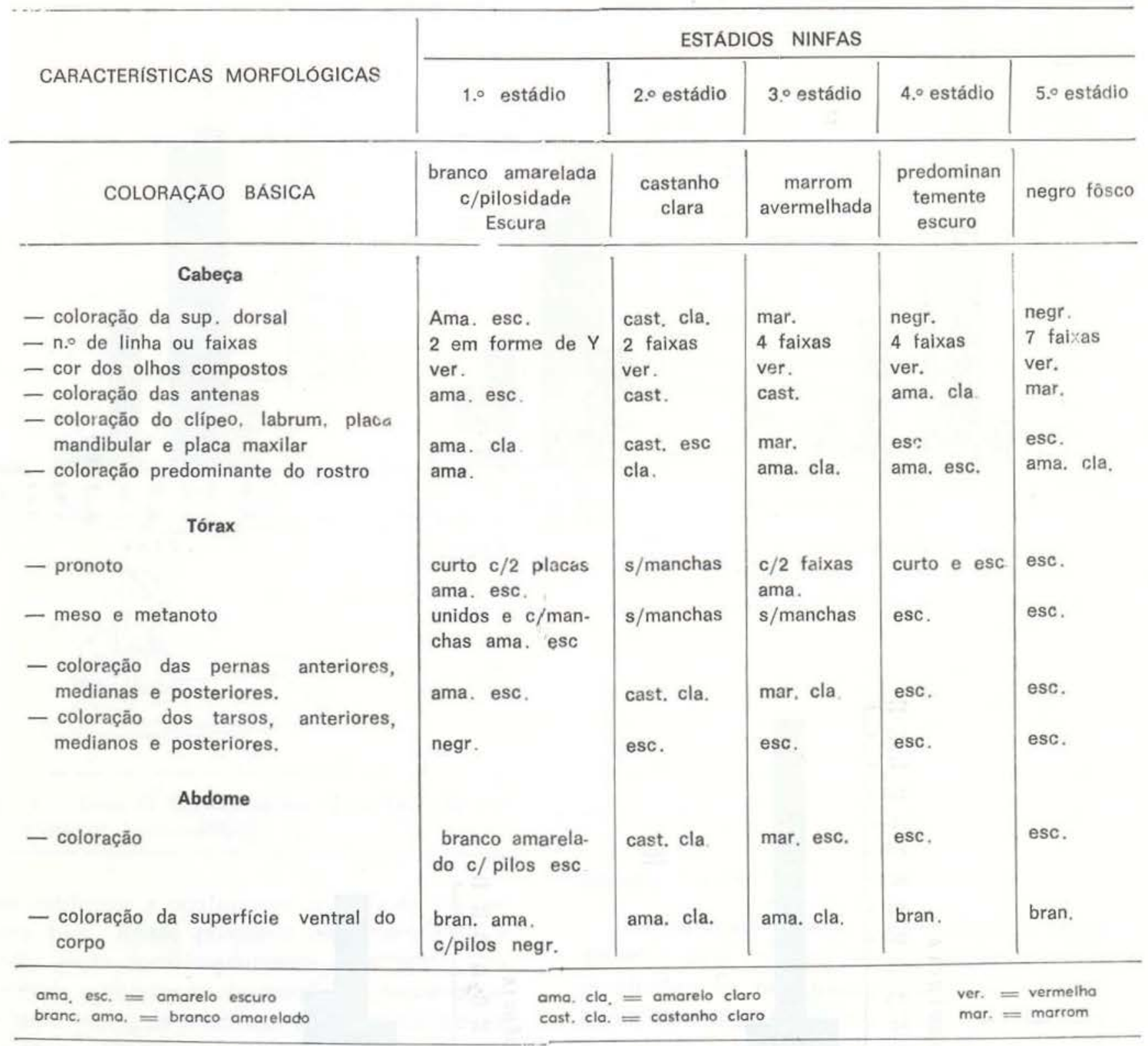

tes nos diferentes estádios, foram mais devidas a acidentes de ecdise e de manuseio dos copos. Na tabela 5 são apresentacios os dados sobre a mortalidade de $L$. recurvus no labora. tório.

\section{DISCUSSÄO}

O gênero Limnogonus é muito semelhante a outros gêneros da família Gerridae. O segmento başal curto do tarso da perna anterior, antenas curtas e fortes com a superfície dorsal da cabeça e pronoto brilhante, são os caracteres chaves para separar o gênero Limnogonus dos gêneros Gerris e Tachygerris (Drake \& Harris, 1934). Neste trabalho, as dificuldades para separarem-se estes gêneros entre si morfologicamente não ocorreram em virtude dos mesmos ocuparem habitat diferentes. Não foram encontrados ainda representantes do gênero Gerris na Amazônia Brasileira. O gênero Tachygerris ocorre em poças de água de chuva e pequenos igarapés no interior de floresta primária; são de difícil captura em virtude de serem velozes no vôo. Na Amazônia, a grande dificuldade de identificar o gênero e em especial a espécie deste estudo, Limnogonus recurvus, está relacionada com as formas imatu- 

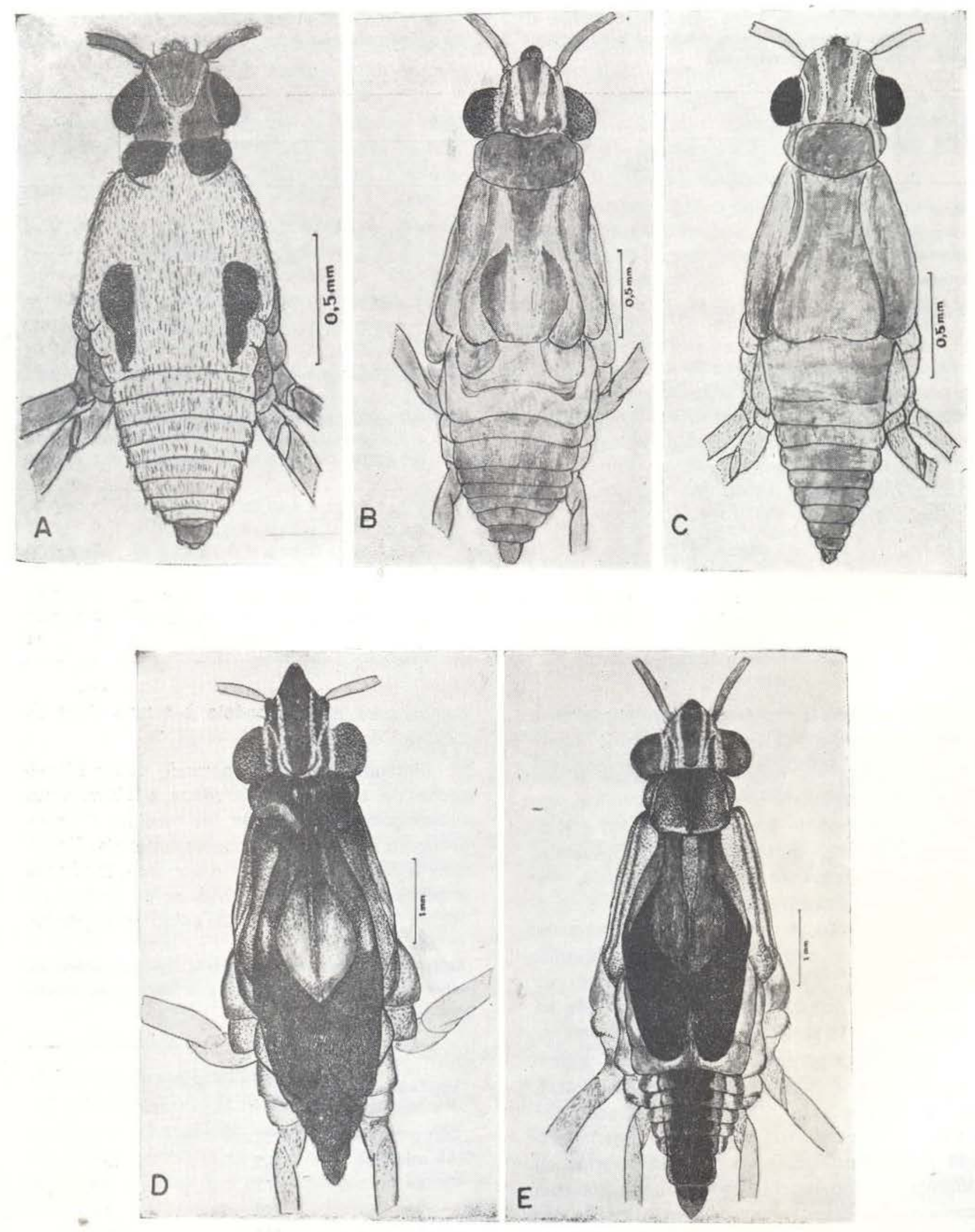

Fig. 3 - Estádios ninfais de L. recurvus: A $-1.0^{\circ}$ estádio; $B-20^{\circ} ; \mathrm{C}-3.0^{\circ}$ D $-4 .^{\circ}$ e $\mathrm{E}-5.0^{\circ}$. 
TABELA 4 - Médias e desvio padrão das medidas $(\mathrm{mm})$ do comprimento total do corpo, largura da fronte, segmen. tos antenais, pernas anteriores, medianas e posteriores de L. recurvus. Medidas para 10 indivíduos de cada estádio feitas com ocular micrometrada.

\begin{tabular}{|c|c|c|c|c|c|c|c|}
\hline \multirow{2}{*}{$\begin{array}{l}\text { Estruturas do corpo } \\
\text { medidas }\end{array}$} & \multicolumn{5}{|c|}{ ESTÁGIOS NINFAIS } & \multirow{2}{*}{$\begin{array}{l}\text { Adulto } \\
\text { fêmea }\end{array}$} & \multirow{2}{*}{$\begin{array}{l}\text { Adulto } \\
\text { macho }\end{array}$} \\
\hline & Primeiro & Segundo & Terceiro & Quarto & Quinto & & \\
\hline \multicolumn{8}{|l|}{ Comprimento Total do } \\
\hline $\begin{array}{l}\text { corpo } \\
\text { Largura da Fronte }\end{array}$ & $\begin{array}{l}1.6 \pm 0.16 \\
0.3 \pm 0.03\end{array}$ & $\begin{array}{l}3.3 \pm 0.54 \\
0.4 \pm 0.05\end{array}$ & $\begin{array}{l}4.2 \pm 0.64 \\
0.6 \pm 0.08\end{array}$ & $\begin{array}{l}5.6 \pm 0.30 \\
0.7 \pm 0.05\end{array}$ & $\begin{array}{l}7.7 \pm 0.49 \\
0.8 \pm 0.03\end{array}$ & $\begin{array}{l}9.9 \pm 0.46 \\
0.8 \pm 0.03\end{array}$ & $\begin{array}{l}9.7 \pm 0.39 \\
0.8 \pm 0.03\end{array}$ \\
\hline \multicolumn{8}{|l|}{ Antenas } \\
\hline 1. ${ }^{\circ}$ segmento & $0.3 \pm 0.02$ & $0.6 \pm 0.10$ & $0.9 \pm 0.13$ & $1.4 \pm 0.05$ & $1.5 \pm 0.16$ & $2.1 \pm 0.13$ & $2.0 \pm 0.08$ \\
\hline 2. ${ }^{\circ}$ segmento & $0.2 \pm 0 \quad 01$ & $0.4 \pm 0.06$ & $0.6 \pm 0.10$ & $1.1 \pm 0.06$ & $1.1 \pm 0.10$ & $1.7 \pm 0.09$ & $1.8 \pm 0.12$ \\
\hline $3 .^{\circ}$ segmento & $0.2 \pm 0.02$ & $0.4 \pm 0.06$ & $0.7 \pm 0.10$ & $1.1 \pm 0.05$ & $1.2 \pm 0.12$ & $1.4 \pm 0.10$ & $1.4 \pm 0.09$ \\
\hline $4 .^{\circ}$ segmento & $0.9 \pm 0.04$ & $1.1 \pm 0.13$ & $1.4 \pm 0.16$ & $1.8 \pm 0.05$ & $1.9 \pm 0.12$ & $2.1 \pm 0.14$ & $2.0 \pm 0.08$ \\
\hline \multicolumn{8}{|l|}{ Pernas Anteriores } \\
\hline Femur & $0.5 \pm 0.06$ & $0.9 \pm 0.15$ & $1.3 \pm 0 \quad 15$ & $2.0 \pm 0.07$ & $2.0 \pm 0.10$ & $2.7 \pm 0.23$ & $2.9 \pm 0.24$ \\
\hline Tíbia & $0.5 \pm 0.03$ & $0.8 \pm 0.14$ & $1.2 \pm 0.15$ & $2.0 \pm 0.06$ & $2.0 \pm 0.09$ & $2.5 \pm 0.06$ & $2.5 \pm 0.13$ \\
\hline Tarso & $0.2 \pm 0.01$ & $0.3 \pm 0.04$ & $0.4 \pm 002$ & $0.6 \pm 0.04$ & $0.7 \pm 0.04$ & $0.8 \pm 0.04$ & $0.8 \pm 0.02$ \\
\hline \multicolumn{8}{|l|}{ Pernas Média } \\
\hline Femur & $1.0 \pm 0.05$ & $2.1 \pm 0.35$ & $3.2 \pm 0.42$ & $5.3 \pm 0.27$ & $5.4 \pm 0.19$ & $7.8 \pm 0.22$ & $7.8 \pm 0.32$ \\
\hline Tíbia & $1.1 \pm 0.02$ & $2.0 \pm 0.29$ & $3.0 \pm 0.43$ & $4.6 \pm 0.22$ & $4.8 \pm 0.15$ & $6.6 \pm 0.40$ & $6.7 \pm 0.30$ \\
\hline Tarso & $0.9 \pm 0.16$ & $1.3 \pm 0.18$ & $1.9 \pm 0.22$ & $2.7 \pm 0.20$ & $2.8 \pm 0.11$ & $3.4 \pm 0.20$ & $3.3 \pm 0.15$ \\
\hline \multicolumn{8}{|l|}{ Pernas Posteriores } \\
\hline Femur & $0.9 \pm 0.05$ & $1.9 \pm 0.55$ & $3.1 \pm 0.46$ & $5.2 \pm 0.25$ & $5.5 \pm 0.18$ & $7.8 \pm 0.22$ & $8.2 \pm 0.39$ \\
\hline Tíbia & $0.5 \pm 0.03$ & $1.0 \pm 0.21$ & $1.6 \pm 0.28$ & $2.6 \pm 0.36$ & $2.8 \pm 0.28$ & $4.5 \pm 0.27$ & $4.4 \pm 0.31$ \\
\hline Tarso & $0.4 \pm 0.02$ & $0.6 \pm 0.06$ & $0.7 \pm 0.10$ & $1.0 \pm 0.00$ & $1.0 \pm 0.03$ & $1.2 \pm 0.10$ & $1.2 \pm 0.14$ \\
\hline
\end{tabular}

ras do gênero Brachymetra no primeiro e segundo estádio. Quanto aos adultos, existe uma certa similaridade com Limnogonus aduncus (Drake \& Harris, 1934).

Os trabalhos de Torre-Bueno (1917) e Hungerford (1919), focalizam de uma maneira simples, aspectos da biologia de Gerris. Semelhanças bastante significativas com a espécie deste estudo, é vista em Gerris fluviorum, cujos ovos são amarelo transparentes quando recentemente eliminados, com $1,70 \mathrm{~mm}$ de comprimento por $0,65 \mathrm{~mm}$ de largura. Não há opérculo e a fenda de eclosão tem a forma de uma cruz. O tempo médio de incubação, à temperatura de $29^{\circ} \mathrm{C}$, foi de 15 dias (Tonapi, 1959). No presente trabalho, foi verificado que os ovos de L. recurvus apresentam uma coloraçāo branco amarelada transparente, forma cilíndrica quando recentemente eliminados com aproximadamente $1 \mathrm{~mm}$ de comprimento por $0,40 \mathrm{~mm}$ de largura. Não há opérculo e a fenda de eclosão tem a forma de uma linha longitudinal. O tempo médio de incubação que verifi- camos para nossa espécie à temperatura de $29^{\circ} \mathrm{C}$ foi de 8 dias.

Matsuda (1961) comparando dados biométricos de Limnogonus aduncus e Limnogonus (Limnogonellus) lubricus, fez medidas do comprimento do corpo, segmentos antenais, fêmur, tíbia e tarsos das pernas anteriores, medianas e posteriores, de 10 indivíduos de ambas espécies. A seguir (Tab. 6) estão relacionados

TABELA 5 - Taxa de mortalidade no laboratório de ninfas de L. recurvus durante seu período de crescimento.

\begin{tabular}{c|c|c}
\hline Estágios ninfais & $\begin{array}{c}\text { N.o de ninfas } \\
\text { observadas }\end{array}$ & $\begin{array}{c}\text { Taxa de mortalidade } \\
\text { em \% }\end{array}$ \\
\hline Ovos eclodidos & 225 & 23,2 \\
$1.0^{\circ}$ & 173 & 3,1 \\
$2.0^{\circ}$ & 166 & 0,0 \\
$30^{\circ}$ & 166 & 0,44 \\
$4 .^{\circ}$ & 165 & 4,44 \\
$5.0^{\circ}$ & 156 & 0.0 \\
\hline
\end{tabular}


apenas a média das 10 medidas de cada estágio de ambas espécies, do comprimento total do corpo, a título de comparação com as medidas de $L$. recurvus.

Comparando-se os dados de Matsuda com os obtidos agora, notamos que apenas as nin. fas de $1 .^{\circ}$ estádio de $L$. recurvus, se assemeIham em tamanho, com as ninfas de $1 .^{\circ}$ estádio de $L$. aduncus, sendo bem maiores do que as ninfas de Limnogonus (L.) lubricus. A descrição das ninfas de ambas as espécies feitas por Matsuda, não apresentam semeihanças significativas com as ninfas de $L$. recurvus.

TABELA 6 - Médias (em $\mathrm{mm}$ ) do comprimento total do corpo de 10 individuos de cada estágio de Limnogonus aduncus e Limnogonus (L) lubricus, segundo Matsuda (1961), comparados com as encontradas para L. recurvus.

\begin{tabular}{c|c|c|c}
\hline Estágios & $\begin{array}{c}\text { Comprimento } \\
\text { total do corpo } \\
\text { de L. } \\
\text { aduncus }\end{array}$ & $\begin{array}{c}\text { Comprimento } \\
\text { total do corpo } \\
\text { p/ L. (L.) } \\
\text { lubricus }\end{array}$ & $\begin{array}{c}\text { Comprimento } \\
\text { total do corpo } \\
\text { P/ L. re- } \\
\text { curvus }\end{array}$ \\
\hline $10^{\circ}$ & 1,6 & 1,2 & 1,6 \\
$2.0^{\circ}$ & 2,3 & 1,6 & 3,3 \\
$3.0^{\circ}$ & 3,2 & 2,2 & 4,2 \\
$4.0^{\circ}$ & 4,1 & 2,3 & 5,6 \\
$5 .^{\circ}$ & 5,4 & 3,7 & 7,7 \\
adulto macho & 8,0 & 3,8 & 9,7 \\
\hline
\end{tabular}

Drake \& Harris (1934), descrevendo L. aduncus, chamou atenção para a grande similaridade que existe entre os adultos desta espécie e os adultos de $L$. recurvus, principalmente nas marcações sobre a superfície dorsal da cabeça. Porém, como é mostrado, por dados biométricos, pode observar-se que há alguma diferença, taivez significativa.

\section{CONCLUSÕES}

Foi comprovado que a criação de L. recurvus no laboratóriơ, dá rendimento satisfatório, quando os insetos são criados individualmentes, pois possibilita um melhor controle dos indivíduos e ocupa um restrito lugar no laboratório.

Segundo a literatura, os ovos de $L$. recurvus são muito semelhantes aos ovos de Gerris fluviorum.
São mostrados pela primeira vez neste trabalho, a descrição das ninfas e dados biométricos de $L$. recurvus.

A oviposição de $L$. recurvus ocorrem em qualquer substrato submerso na água; pode ocorrer em plantas aquáticas, parede das placas ou restos de animias.

É provável que o período de incubação do ovo nesta espécie, não depende somente da temperatura da água, mas também, do $\mathrm{pH}$ e da taxa de oxigênio.

A eclosão do ovo ocorre em aproximada. mente 8 minutos.

$\mathrm{Na}$ tabela 2 estão relacionados os tempos de duração dos estádios ninfais conforme o seguinte: em 173 ninfas de $1 .^{\circ}$ estádio, um tempo médio de duração de 6 dias. Em 166 do $2 .^{\circ}$ estádio, um tempo médio de duraçăo de $51 / 2$ dias, nas 166 do $3 .^{\circ}$ estádio, um tempo médio de duração de $51 / 2$ dias, as 165 do $4 .^{\circ}$ estádio uma média de $61 / 2$ dias e as 156 de $5 .^{\circ}$ estádio, uma média de 9 dias.

A maior taxa de mortalidade no laboratório ocorre em ninfas recem-eclodidas.

\section{SUMMARY}

Somo aspects of the biology of Limnogonus recurvus (Gerridae: Hemiptera) were studied in the laboratory. Adults and nymphs were captured in various types of aquatic habitats, such as: rivers, streams, lakes, and small puddles of rain water. Crosses were made in the laboratory. The cycle from egg to adult is approximately 43 days long.

\section{BIBLIOGRAFIA}

BRINKHURST, R.O.

1959 - A description of the nymphs of British Gerris species (Hemiptera: Heteroptera). Proc. Roy. Entomol. Soc. London, (A) 34 : 130-136.

Bовв, M.L.

1951 - The life history of Gerris canaliculatus Say in Virginia (Hemiptera: Gerridae). Virginia J. Sci., 2:102-108.

CALlahan, J.R.

1974 - Observations on Gerris incognitus and Gerris gillettei (Hoteroptera: Gerridae) Proc. Entomol. Soc. Washington, 76:15-21.

Costa Lima, A.M. dA

1940 - Superfamilia Gerroidea. In: Insetos do Brasil. Rio de Janeiro, Escola Nacional de Agronomia, 2, p. 297-983, 
DARNHOFER-DEMAR, B

1969 - Zur funktions morphologie der wasserläufer. Die morphologie des lokomotions apparates von Gerris lacustris L. Heteroptera: Gerridae) Zool. jb. (Anat.), 86 : 28-66, 9 fig., 2 tab.

DRAKE, C.J.

1954 - An undescribed Metrobates Uhler from Brazil (Hemiptera: Gerridae) Bull. South. Calif. Sci., $53: 50-51$.

1957 - New neotropical Halobatinae (Hemiptera: Gerridae) Bull. Brooklyn ent. Soc., 52: 128-130.

1959 - Three new water-striders from Brazil (Hemiptera: Gerridae) Bull, S. Calif, Acad. Sci. Los Angeles, 58:107-111, 1 fig.

Drake, C.J. \& Chapman, C.H.

1954 - New american waterstriders (Hemiptera) Florida Ent., 37(3) : 151-155.

Drake, C.J. \& Carvalho, J.C.M.

1954 - New waterstriders from Brazil (Hemiptera) Proc. biol. Soc. Washington, 67: 223-226, 2 fig.

DRAKE, C.J. \& HARris, H.M.

1930 - Notes on some South American Gerridae (Hemiptera). Ann. Carneg. Mus., 19: 235-239.

1934 - The Gerrinae of the Western Hemisphere (Hemiptera), Ann. Carneg. Mus., 23: 179-240.

DUFOUR, L.

1833 - Recherches anatomique et philosophiques sue les Hemipteres, accompagnées de considerations relative a I'histoire naturelle et la classification des insectes. Paris (Apud Hungerford, 1919).

HOFFMAN, W.E.

1924 - The life histories of three species of gerrids (Heteroptera: Gerridae). Ann. Entomol. Soc. Am., 7 : 419-430.

HUNGERFORD, H.B.

1919 - The b:ology and ecology of aquatic and semiaquatic Hemiptera. Kanasas Univ. Sci. Bull., $21: 1-328$.

Kenaga, E.

1941 - The genus Telmatometra Bergroth (Hemiptera: Gerridae). Univ. Kansas Sci. Bull., $27: 169-185$.

1942 - A new genus in the Halobatinae (Gerridae: Hemiptera). J. Kansas ent. Soc., 15: 136-141.
KUITERT, L.

1942 - Gerrinae in the University or Kansas collections. Univ. Kansas Sci. Bull., 28 : 125-131.

MATSUDA, R.

1960 - Morphology, Evolution and a Classification of the Gerridae (Hemiptera: Heteroptera). Kansas Univ. Sci. Bull, $41: 24-632$.

1961 - Studies of relative growth in Gerridae (Hemiptera: Heteroptera). J. Kansas ent. Soc., $34: 5-17,5$ fig.

PENN, C.H. \& GoldSMITH, R.M.

1950 - The life history of the Southern waterstrider, Gerris conalicullatus (Hemiptera: Gerridae). J. Tenn. Acad. Sci., 25 : 76-79.

PoIsson, R

1922 - Armature génitale et structurs chitineuse du pénis dans la genre Gerris (Hem. Hidrometridae). Bull. Soc. Ent. France, 12: 171-173.

1957 - Faune de France 61. Hétéroptères aquatiques. Féd. France Soc. Sci. Nat. (P. Lechavalier, Paris), 263 p (larves des Gerris), p. $245-251$.

1965 - Catalogue des insectes hétéroptères Gerridae Leach, 1807, africano-malgaches. Bull. Inst. franç. Afr. noire, Dakar (A), 27 : 1466-1503.

SCHROEDER, H.O.

1931 - The genus Rheumatobates and notes on the male genitalia of some Gerridae (Hemiptera: Gerridae) Kansas Univ. Sci. Bull., $20: 63-69,6$ fig.

Shaw, G.J.

1933 - A study of the genus Brachymetra ( $\mathrm{He}$ miptera: Gerridae). Univ. Kansas Sci. Bull. $21: 221-233$.

SPRAGUe, I.B.

1967 - Nymphs of the genus Gerris in New England. Ann. Entomol. Soc. Amer., 60: 1038-1044.

TONAPI, G.T.

1959 - A note on the eggs of Gerris fluviorus $F$. with a brief description of the neanidae (Hemiptera: Gerridae). Ent. Mon. Mag. London, $95: 29-31,6$ fig.

TORRE-BUENO, J.R.

1917 - Life history and habits of Gerris remiges Say (Hem.). Entomol. News, 28 : 201-208.

(Aceito para publicação em 19/06/79) 